\title{
Mechanistic insights into chiral proofreading during translation of genetic code
}

Satya Brata Routh ${ }^{1}$, Komal Ishwar Pawar ${ }^{1}$, Katta Suma ${ }^{1}$, Mantu Kumar ${ }^{1}$, Shobha P. Kruparani ${ }^{1}$, Rajan Sankaranarayanan ${ }^{1}$

${ }^{1}$ CSIR-Centre For Cellular And Molecular Biology, Hyderabad, India

E-mail: satya_b@ccmb.res.in

Exclusion of D-amino acids from the translational apparatus is essential for protein homeostasis and cellular integrity. An enzyme named D-aminoacyl-tRNA deacylase (DTD) decouples D-amino acids that have been mischarged on tRNAs, thereby preventing cellular toxicity due to D-amino acids [1]. DTD has thus been implicated as a key cellular chiral checkpoint during protein biosynthesis. Using ligand-bound crystal structures in conjunction with mutational and biochemical analyses, we have deciphered the mechanisms of enantioselectivity and catalysis of this chiral proofreading enzyme. DTD is extremely enantioselective because it does not act on even the smallest amino acid with L-chirality, i.e., L-alanine [2,3]. It employs an invariant, cross-subunit Gly-cisPro motif to ensure stereospecificity during substrate selection. The rigid fixation of the cis conformation of the motif disposes the two carbonyl oxygens in a near parallel orientation, which forms the structural basis of DTD's enantioselectivity. Any perturbation of the motif renders the enzyme inactive, thereby suggesting its importance in the maintenance of the active site architecture [2]. However, the enzyme functions only through strict L-chiral rejection and not D-chiral selection. DTD's chiral proofreading site is therefore porous to achiral glycine which in turn leads to glycine "misediting paradox". Nevertheless, elongation factor thermo unstable (EF-Tu) protects the achiral substrate Gly-tRNAGly against DTD's unwarranted activity [3]. On the basis of ligand-bound crystal structures of a structural homolog of DTD, we had proposed that DTD uses the $2^{\prime}-\mathrm{OH}$ of A76 of tRNA for catalysis. Our mutational studies later demonstrated that active site residues of DTD are dispensable for catalysis [2]. Using 2'-modified tRNAs, we have recently shown that DTD employs the $2^{\prime}-\mathrm{OH}$ to effect hydrolysis of its substrates [2]. Thus, DTD is a primordial RNA-based enzyme which functions at the RNA-protein interface.

[1] Soutourina, O. et al. (2004). J. Biol. Chem. 279:42560-42565.

[2] Ahmad, S., Routh, S. B. et al. (2013). Elife, 2:e01519, 1-18.

[3] Routh, S. B. et al. (2016). PLoS Biol. 14:e1002465, 1-22.

Keywords: translation of genetic code, RNA-based catalysis, chiral proofreading/editing 